\title{
Modeling and Stability Analysis of Host-parasite Population Dynamics
}

\author{
Geremew Kenassa Edessa, Purnachandra Rao Koya \\ Department of Mathematics, Wollega University, Nekemte, Ethiopia \\ Email address: \\ gbonsa.kena@gmail.com(G. K. Edessa),drkpraocecc@yahoo.co.in (P. R. Koya)
}

\section{To cite this article:}

Geremew Kenassa Edessa, Purnachandra Rao Koya. Modeling and Stability Analysis of Host-parasite Population Dynamics. Mathematical Modelling and Applications. Vol. 5, No. 2, 2020, pp. 118-128. doi: 10.11648/j.mma.20200502.17

Received: January 1, 2020; Accepted: May 5, 2020; Published: May 28, 2020

\begin{abstract}
In this study, a mathematical model is developed to explore the population dynamics of two host species. Both the hosts depend on the same resources and the availability of such resources is limited in nature. If the host populations increase abnormally the limited natural resources will be used up. Hence, the concept of parasite is brought in to the picture to regulate the host populations. The parasite is a mechanism that reduces the host populations. However, on one hand if the parasite attacks more the hosts may extinct and on the other hand if the parasite do not attack then the host populations may increase and resource may be used up. Hence, the parasite is expected to maintain a balance so that neither the host populations nor the resources extinct. Here, both the hosts are classified in to susceptible and infected and hence the model comprises of four populations: Susceptible Host-1, Infected Host-1, Susceptible Host -2 and Infected Host-2. Thus, the mathematical model comprises of a system of four first order non-linear ordinary differential equations. Mathematical analysis of the model is conducted. Positivity and boundedness of the solution have been verified and thus shown that the model is physically meaningful and biologically acceptable. Equilibrium points of the model are identified and stability analysis is conducted. Simulation study is conducted in order to support the mathematical analysis using software packages Mat lab and DeDiscover.
\end{abstract}

Keywords: Modeling, Hosts, Parasite-mediated Interactions, Stability, Numerical Simulation

\section{Introduction}

Parasites can theoretically encourage indirect interactions among ecological communities those live in the same trophic level. These interactions lead to some kind of effects on the competing species. Such interactions are also called as parasite-mediated competition [1-2, 4, 13-15, 19]. However, parasites induce competition-like indirect interactions among species that would not otherwise happen at all $[5,8]$. These ideas reflect indirect effects of parasites on community composition leading to coexistence or promoting exclusion.

\subsection{Parasite-mediated Competition}

Parasites may affect competitive dynamics between native and introduced animals and plants through density and trait effects [3, 6, 13-15].

Models of parasite-mediated competition have provided insight into the role of shared parasites in shaping communities of competing species. Parasites can regulate the population density of their hosts and, as a result, affect the dynamics of the community, and can cause the extinction of local populations and of whole species [2, 3, 13-15].

In this present study, parasites are predicted to enhance the range of conditions leading to coexistence if, for example, one of the host are more heavily impacted by the parasite. However, some arguments argued that the predominant pressure exerted by parasites on communities might not be the result of catastrophic outbreaks, but rather of less virulent and persistent where the parasites moderate or enhance the competitive capabilities of their hosts [3, 13].

When viewed as part of an ecosystem, the parasitized host is subject to numerous other interactions that convey benefits, no apparent benefits, or harm or not benefits to it $[11,12,5$, 20]. Argued that the direct outlay to the host that reduce its fitness can be either aggravated by indirect consequences (i.e. where parasitism renders predation more likely), or improved by indirect consequences. 


\subsection{Apparent Competition}

In the context of parasitism, apparent competition is predicted to occur when two species that do not otherwise interact both host with the same parasite species [16-18, 22]. As both host species are a resource for the parasite, population density increases in either host lead to reductions in the other, via the, density mediated, negative effects of the parasite. Apparent competition can theoretically lead to the elimination of one host, indirectly coupling the dynamics of host species with different habitat or resource requirements.

In view of these, this study targeted to present these synthesized empirical and theoretical work using mathematical modeling and analysis qualitatively how parasites influences competitive and predatory interactions between species $[1,7,10,13,21,23]$. We highlight the sensitive parameters to assess the impact of parasites on communities by incorporating the theoretical and empirical studies so as to examine how the effects of parasitism. Moreover, parasitism scales up the community-level processes.

In this paper, comparison of the model system would be carried out through the investigation of the interaction made between the populations directly and indirectly to each other and with parasite.

\section{Assumption and Model Formulation}

Accordingly, Mathematical epidemiology to study the dynamics of diseases spread has become an interesting topic of research study and received much attention from scientists after the pioneering work of [2, 3, 13-15, 20].

In this recent study, by applying a deterministic framework of an ecological model of the infection of parasite, the analysis of the population dynamics of eco-epidemiology would be investigated.

To start with, the mathematical model of a single type of host and its parasite would be defined by considering the following descriptions. These are the changes in the numbers of hosts $H$ and free-living parasite. These changes are due to three minimum processes: host birth, mortality due to parasite infection and natural death of a host. Hence, the classical model of the combination of host and parasite population growth with carrying capacity of host which follows the general deterministic trajectories defined as

$$
\begin{aligned}
& \frac{d H}{d t}=r H\left(1-\frac{H}{k}\right)-\delta H P \\
& \frac{d P}{d t}=\delta m H P-d P ;
\end{aligned}
$$

This model is shown with simple diagram in figure 1 . This system is similar to a predator-prey system but differing by the quantity $m$ and called in host-parasite system burst size where as in predator-prey system ecological efficiency. Regardless of the potentially widespread effects of parasites on the community, most studies of host-parasite dynamics focus on the effects of a single parasite on a single host, neglecting the host's interactions with each other and with other species.

The present model considers interaction of two hosts. These hosts do not directly interact with each other but they are mediated by a common enemy or parasite.

In this paper, the recovery of the hosts would not be considered. Moreover, in this study the interactions involving parasites that feed on a host individual usually living on or in it and often causing harm but not immediate death and the carrying capacity is only allowed for the uninfected hosts. i.e., for infected hosts limited resources are available. Further, responses of the hosts are linear rather than saturated. In this assumption, the model for free living parasite is not explicitly modeled.

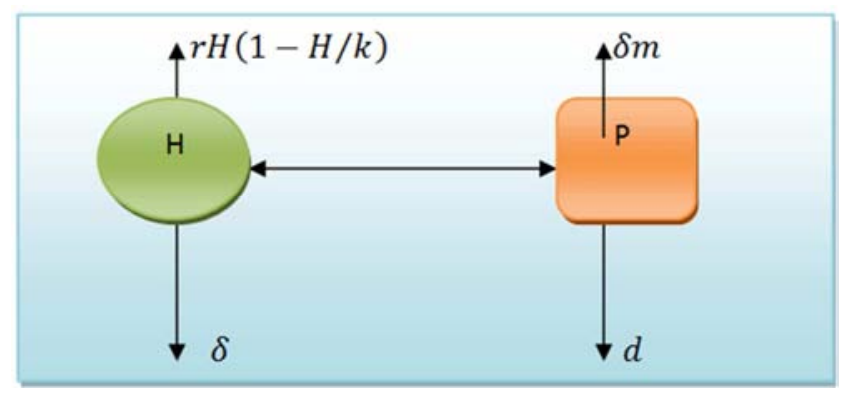

Figure 1. Simple schematic diagram of system (1) and (2).

The interaction between the states of the present model is illustrated in Figure 2 below;

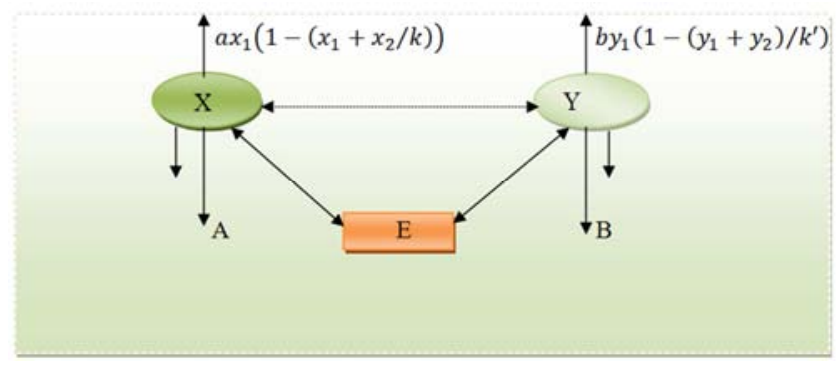

Figure 2. Schematic diagrams of the population dynamics.

The present mathematical model is formulated based on the above schematic model, and is given by the following system of differential equation:

$$
\begin{aligned}
& \frac{d x_{1}}{d t}=a x_{1}\left(1-\frac{x_{1}+x_{2}}{k}\right)-\mu x_{1} x_{2}-\alpha x_{1} \\
& \frac{d x_{2}}{d t}=\mu x_{1} x_{2}-A x_{2} ; A=\alpha+\delta \\
& \frac{d y_{1}}{d t}=b y_{1}\left(1-\frac{y_{1}+y_{2}}{k^{\prime}}\right)-r y_{1} y_{2}-\theta y_{1} \\
& \frac{d y_{2}}{d t}=r y_{1} y_{2}-B y_{2} ; B=\beta+\theta
\end{aligned}
$$

Here, $X(t)=x_{1}(t)+x_{2}(t)$ and $Y(t)=y_{1}(t)+y_{2}(t)$ denote total population sizes of the first and second hosts respectively. However, the total size of both the hosts in the system is given by $W(t)=X(t)+Y(t)$.

Descriptions of notations, parametric values of the system 
are given in the following tables.

Table 1. Notations and descriptions of the model variables.

\begin{tabular}{ll}
\hline Variables & Descriptions \\
\hline$x_{1}(t)$ & Density of first susceptible host population \\
$x_{2}(t)$ & Density of first infectious host population \\
$y_{1}(t)$ & Density of second susceptible host population \\
$y_{2}(t)$ & Density of second infectious host population \\
\hline
\end{tabular}

Table 2. Notations and descriptions of the parameters used in the model.

\begin{tabular}{ll}
\hline Parameters & Descriptions \\
\hline$a$ & Growth rate of host-1 \\
$b$ & Growth rate of host-2 \\
$\mu$ & Infection transmission rate of host-1 \\
$r$ & Infection transmission rate of host-2 \\
$\alpha$ & Natural death rate of host-1 \\
$\theta$ & Natural death rate of host-2 \\
$\beta$ & Mortality rate due to disease of host-2 \\
$A$ & Total death rate of host-1 \\
$B$ & Total death rate of host-2 \\
$k$ & Environmental carrying capacity for host-1 \\
$k^{\prime}$ & Environmental carrying capacity for host-2 \\
\hline
\end{tabular}

\section{Qualitative Analysis of the Model Systems}

\subsection{Positivity and Boundedness of the System}

For the model to be meaningful and well posed, it is necessary that the solutions of (3)-(6) with positive initial data are positive and bounded for all $t>0$. This fact has been stated and proved in the following:

Proposition 1: All solutions of the model equations (3)-(6) are non-negative and bounded.

Proof:

Positivity of the populations: consider the equation of system (3) - (6).

Up on integrating the model equations (3)-(6) with respect to $t$ the analytical solutions of the model variables are obtained as

$$
\begin{gathered}
x_{1}(t)= \\
x_{01} \exp \int_{0}^{t}\left\{a\left(1-\frac{x_{1}(s)+x_{2}(s)}{k}\right)-\mu x_{2}(s)-\alpha\right\} d s \\
x_{2}(t)=x_{02} \exp \int_{0}^{t}\left\{\mu x_{1}(q)-A\right\} d q \text { Host }-1 \\
y_{1}(t)= \\
y_{01} \exp \int_{0}^{t}\left\{b\left[1-\frac{y_{1}(u)+y_{2}(u)}{k^{\prime}}\right]-r y_{2}(u)-\theta\right\} d u \text { Host }-2 \\
y_{2}(t)=y_{02} \exp \int_{0}^{t}\left\{r y_{1}(v)-B\right\} d v
\end{gathered}
$$

Here, it can be observed that each solution is a product of an initial condition and an exponential function. However, by definition every initial condition and exponential function are non-negative quantities, so are their products.
Therefore, all solutions of the system of equations (3)-(6) are non-negatives for all $t \geq 0$.

Boundedness of the system

In theoretical eco-epidemiology, a system is bounded implies that the system is biologically valid and well behaved. Here in the present study, the biological validity of the model is achieved by showing that the solutions of (3)-(6) are bounded.

In order to show the system is bounded, let the expression for the total population of the system as $W(t)=x_{1}(t)+$ $x_{2}(t)+y_{1}(t)+y_{2}(t)$ be considered. Also, let $m W=$ $m\left(x_{1}+x_{2}+y_{1}+y_{2}\right) \quad$ and $\quad(d W / d t)=\left(d x_{1} / d t\right)+$ $\left(d x_{2} / d t\right)+\left(d y_{1} / d t\right)+\left(d y_{2} / d t\right)$ where $m$ is any positive constant. Now, using the fore going expressions in the addition of (3)-(6) reduces the result as:

$$
\begin{gathered}
\frac{d W}{d t}+m W=a x_{1}\left(1-\frac{x_{1}+x_{2}}{k}\right) \\
+b y_{1}\left(1-\frac{y_{1}+y_{2}}{k^{\prime}}\right)-\left(\alpha x_{1}+A x_{2}+\theta y_{1}+B y_{2}\right)+m W \\
\frac{d W}{d t}+m W \leq a x_{1}\left(1-\frac{x_{1}+x_{2}}{k}\right)+b y_{1}\left(1-\frac{y_{1}+y_{2}}{k^{\prime}}\right)+m x_{1}+ \\
m y_{1}, \text { For } A \text { and } B>m \\
=(a+m) x_{1}-a \frac{x_{1}^{2}}{k}-a \frac{x_{1} x_{2}}{k}+(b+m) y_{1}-b \frac{y_{1}^{2}}{k^{\prime}}-b \frac{y_{1} y_{2}}{k^{\prime}} \\
\leq(a+m) x_{1}-a\left(x_{1}^{2} / k\right)+(b+m) y_{1}-b\left(y_{1}^{2} / k^{\prime}\right) \\
=\frac{k}{4 a}(a+m)^{2}-\frac{a}{k}\left[x_{1}-\frac{k}{2 a}(a+m)\right]^{2}+\frac{k^{\prime}}{4 b}(b+m)^{2} \\
-\frac{b}{k^{\prime}}\left[y_{1}-\frac{k^{\prime}}{2 b}(b+m)\right]^{2} \\
\leq(k / 4 a)(a+m)^{2}+\left(k^{\prime} / 4 b\right)(b+m)^{2}
\end{gathered}
$$

Now, introducing notation $\omega=(k / 4 a)(a+m)^{2}+$ $\left(k^{\prime} / 4 b\right)(b+m)^{2}$ the differential inequality reduces to the form $(d W / d t)+m W \leq \omega$. It is a first order ordinary differential equation with constant coefficients and its analytical solution is given by $W(t) \leq(\omega / m)(1-$ $\left.e^{-m t}\right)+W_{0} e^{-m t}$. However, in the limit as $t \rightarrow \infty$, the solution takes the form as $W(t) \leq(\omega / m)$ showing that the total population size is bounded. Hence, each population size is also bounded. Thus, the statement holds true.

\subsection{Existence of Steady States of the Model}

The model equations (3)-(6) possess equilibrium points which are biologically feasible. These are obtained by setting $\left(d x_{1} / d t\right)=\left(d x_{2} / d t\right)=\left(d y_{1} / d t\right)=\left(d y_{2} / d t\right)=0 \quad$ and solving the resultant expressions. Thus, the nine equilibrium points of the model and the corresponding existence conditions are listed as follows:

(1) Trivial equilibrium $\mathrm{E}_{0}=(0,0,0,0)$ alwaysexists. At this all the populations are absent.

(2) Axial equilibrium $\mathrm{E}_{1}=\left(\mathrm{x}_{1}^{*}, 0,0,0\right)$ exists if a $>\alpha$. Here, only susceptible host -1 is present.

(3) Axial equilibrium $E_{2}=\left(0,0, y_{1}^{*}, 0\right)$ exists 
if $b>\theta$. Here, only susceptible host -2 is present.

(4) Disease free equilibrium $E_{3}=\left(x_{1}^{*}, 0, y_{1}^{*}, 0\right)$ exists. At this equilibrium both susceptible host-1 and host-2 are present.

(5) Equilibrium $\mathrm{E}_{4}=\left(\mathrm{x}_{1}^{* *}, \mathrm{x}_{2}^{* *}, 0,0\right)$ exists if $\mathrm{a}[1-$ $(\mathrm{A} / \mu \mathrm{k})]>\alpha$ and $\mu \mathrm{k}>A$. Here both susceptible and infected populations of host-1 are present.

(6) Equilibrium $\mathrm{E}_{5}=\left(0,0, \mathrm{y}_{1}^{* *}, \mathrm{y}_{2}^{* *}\right)$ exists if $\mathrm{b}[1-$ $\left.\left(\mathrm{B} / \mathrm{rk}^{\prime}\right)\right]>\theta$ and $\mathrm{rk}^{\prime}>B$. Here both susceptible and infected populations of host-2 only exist.

(7) Equilibrium $\mathrm{E}_{6}=\left(\mathrm{x}_{1}^{*}, 0, \mathrm{y}_{1}^{* *}, \mathrm{y}_{2}^{* *}\right)$ exists. This is a disease-free equilibrium of host-1.

(8) Equilibrium $\mathrm{E}_{7}=\left(\mathrm{x}_{1}^{* *}, \mathrm{x}_{2}^{* *}, \mathrm{y}_{1}^{*}, 0\right)$ exists. This is a disease-free equilibrium of host-2.

(9) Equilibrium $E_{8}=\left(x_{1}^{* *}, x_{2}^{* *}, y_{1}^{* *}, y_{2}^{* *}\right)$ exists. This is a co-existence equilibrium i.e., all types of hosts present here.

Here in the equilibrium points some notations are used in the coordinates those represent the following expressions:

$$
\begin{aligned}
\mathrm{x}_{1}^{*}=[\mathrm{k}(\mathrm{a}-\alpha) / \mathrm{a}], & \mathrm{x}_{1}^{* *}=(\mathrm{A} / \mu), \mathrm{x}_{2}^{* *} \\
= & {[\mathrm{k} /(\mu \mathrm{k}+\mathrm{a})][\mathrm{a}-\alpha-(\mathrm{aA} / \mu \mathrm{k})], }
\end{aligned}
$$

$$
\begin{gathered}
\mathrm{y}_{1}^{*}=\left[\mathrm{k}^{\prime}(\mathrm{b}-\theta) / \mathrm{b}\right], \mathrm{y}_{1}^{* *}=\mathrm{B} / \mathrm{r}, \mathrm{y}_{2}^{* *}=\left[\mathrm{k}^{\prime} /\left(\mathrm{b}+\mathrm{rk}^{\prime}\right)\right][\mathrm{b}- \\
\left.\theta-\left(\mathrm{bB} / \mathrm{rk}^{\prime}\right)\right] .
\end{gathered}
$$

\subsection{Variational Matrix of the Model Equations}

Set the model equations (3)-(6) as

$$
\begin{aligned}
& d x_{1} / d t=f\left(x_{1}, \quad x_{2}, \quad y_{1}, \quad y_{2}\right), \\
& M=\left[\begin{array}{cc}
a\left(1-\frac{2 x_{1}+x_{2}}{k}\right)-\mu x_{2}-\alpha & -\frac{a x_{1}}{k}-\mu x_{1} \\
\mu x_{2} & \mu x_{1}-A \\
0 & 0 \\
0 & 0
\end{array}\right.
\end{aligned}
$$

\subsection{Stability Analysis of Model System}

\subsubsection{Local Stability of the System}

Recall that,

(i) An equilibrium point is said to stable if the variational matrix at that equilibrium has all negative eigenvalues,

(ii) An equilibrium point is said to unstable if the variational matrix at that equilibrium has at least one positive eigenvalues,

This fact is used in verifying whether a given equilibrium is stable or unstable. Here, Local stability analysis of the system at the equilibrium points is conducted and the results are presented in form of theorems and proofs in what follows:

Theorem 1The trivial equilibrium point $\mathrm{E}_{0}=(0,0,0,0)$ is unstable.

Proof:

The variational matrix $M$ at the equilibrium point $\mathrm{E}_{0}$ is denoted by $M_{0}$ and is given by

$$
\begin{aligned}
& d x_{2} / d t=g\left(x_{1}, \quad x_{2}, \quad y_{1}, \quad y_{2}\right), \\
& d y_{1} / d t=h\left(x_{1}, \quad x_{2}, \quad y_{1}, \quad y_{2}\right), \\
& d y_{2} / d t=k\left(x_{1}, \quad x_{2}, \quad y_{1}, \quad y_{2}\right)
\end{aligned}
$$

The variational matrix is defined as

$$
M=\left(\begin{array}{llll}
\frac{\partial f}{\partial x_{1}} & \frac{\partial f}{\partial x_{2}} & \frac{\partial f}{\partial y_{1}} & \frac{\partial f}{\partial y_{2}} \\
\frac{\partial g}{\partial x_{1}} & \frac{\partial g}{\partial x_{2}} & \frac{\partial g}{\partial y_{1}} & \frac{\partial g}{\partial y_{2}} \\
\frac{\partial h}{\partial x_{1}} & \frac{\partial h}{\partial x_{2}} & \frac{\partial h}{\partial y_{1}} & \frac{\partial h}{\partial y_{2}} \\
\frac{\partial k}{\partial x_{1}} & \frac{\partial k}{\partial x_{2}} & \frac{\partial k}{\partial y_{1}} & \frac{\partial k}{\partial y_{2}}
\end{array}\right)
$$

Now, the elements of the matrix $M$ are computed as:

$$
\begin{gathered}
\partial f / \partial x_{1}=a\left\{1-\left[\left(2 x_{1}+x_{2}\right) / k\right]\right\}-\mu x_{2}-\alpha, \partial f / \partial x_{2}= \\
-\left[\left(a x_{1} / k\right)+\mu x_{1}\right], \\
\partial f / \partial y_{1}=\partial f / \partial y_{2}=0, \partial g / \partial x_{1}=\mu x_{2}, \partial g / \partial x_{2}=\mu x_{1}-A, \\
\partial g / \partial y_{1}=\partial g / \partial y_{2}=0, \partial h / \partial x_{1}=\partial h / \partial x_{2}=0, \partial h / \partial y_{1} \\
=b\left\{1-\left[\left(2 y_{1}+y_{2}\right) / k^{\prime}\right]\right\}-r y_{2}-\theta \\
\partial h / \partial y_{2}=-\left[\left(b y_{1} / k^{\prime}\right)+r y_{1}\right], \partial k / \partial x_{1}=\partial k / \partial x_{2}= \\
0, \partial k / \partial y_{1}=r y_{2}, \partial k / \partial y_{2}=r y_{1}-B .
\end{gathered}
$$

Thus, the variational matrix takes the form as

$$
\begin{aligned}
& \begin{array}{ll}
0 & 0
\end{array} \\
& 00 \\
& \left.\begin{array}{c}
b\left(1-\frac{2 y_{1}+y_{2}}{k^{\prime}}\right)-r y_{2}-\theta-\frac{b y_{1}}{k^{\prime}}-r y_{1} \\
r y_{2} \quad r y_{1}-B
\end{array}\right] \\
& M_{0}=\left[\begin{array}{cccc}
a-\alpha & 0 & 0 & 0 \\
0 & -A & 0 & 0 \\
0 & 0 & b-\theta & 0 \\
0 & 0 & 0 & -B
\end{array}\right]
\end{aligned}
$$

Its characteristic equation defined by $\operatorname{det}\left(M_{0}-\lambda I\right)=0$ takes the form as $(a-\alpha-\lambda)(-A-\lambda)(b-\theta-\lambda)(-B-$ $\lambda)=0$. On solving which eigenvalues of $M_{0}$ are obtained as $\lambda_{1}=a-\alpha, \lambda_{2}=-A, \lambda_{3}=b-\theta, \lambda_{4}=-B$. Here, $\lambda_{1}$ and $\lambda_{3}$ are positive eigenvalues since the parameters satisfy the relations $a>\alpha$ and $b>\theta$. That is, here at least one eigenvalues is positive. Hence, the equilibrium point $\mathrm{E}_{0}$ is unstable.

Theorem 2The disease-free equilibrium point $E_{1}=$ $\left(x_{1}{ }^{*}, 0, y_{1}{ }^{*}, 0\right)$ is stable if the following conditions on the parameters hold true: $[(A / \mu k)+(\alpha / a)]>1$ and $\left[\left(B / r k^{\prime}\right)+(\theta / b)\right]>1$.

Proof:

The variational matrix $M$ at the disease-free equilibrium point $\mathrm{E}_{1}$ is denoted by $M_{1}$ and given by: 


$$
M_{1}=\left[\begin{array}{cccc}
\alpha-a & 0 & 0 & 0 \\
0 & \frac{\mu k(a-\alpha)}{a}-A & 0 & 0 \\
0 & 0 & \theta-b & 0 \\
0 & 0 & 0 & -B
\end{array}\right]
$$

Its characteristic equation is given bydet $\left(M_{1}-\lambda I\right)=0$. On solving which the eigenvalues of $M_{1}$ are obtained as $\quad \lambda_{1}=\alpha-a, \lambda_{2}=\{[\mu k(a-\alpha) / a]-A\}, \lambda_{3}=$ $(\theta-b), \lambda_{4}=\left[r k^{\prime}(b-\theta) / b\right]-B$. Clearly, $\lambda_{1}$ and $\lambda_{3}$ are negative values. But, $\lambda_{2}$ and $\lambda_{4}$ are negative quantities if the conditions $[(A / \mu k)+(\alpha / a)]>1$ and $\left[\left(B / r k^{\prime}\right)+(\theta / b)\right]>$ 1 are satisfied. Hence, the equilibrium point $E_{1}$ is stable under the considered assumption.

Theorem 3The equilibrium point $E_{2}=\left(0,0, y_{1}{ }^{*}, y_{2}{ }^{*}\right)$ is stable if $Q^{2}-4 K>0$.

Proof:

The variational matrix $M$ at equilibrium $\mathrm{E}_{2}$ is denoted by $M_{1}$ and given by

$$
M_{2}=\left[\begin{array}{cccc}
a-\alpha & 0 & 0 & 0 \\
0 & -A & 0 & 0 \\
0 & 0 & \left(b-\theta-b B / r k^{\prime}\right)\left(r k^{\prime} / b+r k^{\prime}\right)-b B / r k^{\prime} & -B / r\left(\mathrm{~b}+\mathrm{rk}^{\prime} / \mathrm{b}\right) \\
0 & 0 & \left(r k^{\prime} / b+r k^{\prime}\right)\left(b-\theta-b B / r k^{\prime}\right) & 0
\end{array}\right]
$$

Its characteristic equation is given by $\operatorname{det}\left(M_{2}-\lambda I\right)=0$. By solving the eigenvalues of $M_{2}$ becomes $\lambda_{1}=\alpha-a, \lambda_{2}=$ $-A, \lambda_{3}=(1 / 2)\left[-Q+\sqrt{Q^{2}-4 K}\right], \lambda_{4}=(1 / 2)[-Q-$ $\left.\sqrt{Q^{2}-4 K}\right] \quad$ where $Q=$ $\left[b-\theta-\left(b B / r k^{\prime}\right)\right]\left[r k^{\prime} /\left(b+r k^{\prime}\right)\right]-\left(b B / r k^{\prime}\right), K=$ $(B / b)\left[\theta-b+\left(b B / r k^{\prime}\right)\right]$. It can be observed that both eigenvalues $\lambda_{1}$ and $\lambda_{2}$ are negative quantities. Also, the values $\lambda_{3}$ and $\lambda_{4}$ are also negatives if both the conditions $Q^{2}-$ $4 K>0$ and $K>0$ hold true simultaneously.

Thus, equilibrium point $E_{2}$ is stable under the considered condition.

Theorem 4The equilibrium point $E_{3}=\left(x_{1}{ }^{*}, x_{2}{ }^{*}, 0,0\right)$ is stable if $b<\theta$ and $H>$ 1otherwise unstable.

Proof:

Consider

$$
M_{3}=\left[\begin{array}{cccc}
(-a A / \mu k) & -A(a+k \mu) / \mu & 0 & 0 \\
(\mu k / \mu k+a)(a-\alpha-a A / \mu k) & 0 & 0 & 0 \\
0 & 0 & b-\theta & 0 \\
0 & 0 & 0 & -B
\end{array}\right] \text {, }
$$

But applying the elementary linear algebra on interchanging column operation this $\mathrm{M}_{3}$ is equivalent to;

$$
M_{3}^{\prime}=\left[\begin{array}{cccc}
-A(a+k \mu) / \mu & (-a A / \mu k) & 0 & 0 \\
0 & (\mu k / \mu k+a)(a-\alpha-a A / \mu k) & 0 & 0 \\
0 & 0 & b-\theta & 0 \\
0 & 0 & 0 & -B
\end{array}\right]
$$

Now, the variational matrix at equilibrium $E_{3}$ is equivalently denoted by $M_{3}$ 'and its characteristic equation is given bydet $\left(M_{3}{ }^{\prime}-\lambda I\right)=0$. This gives the eigenvalues

$$
\lambda_{1}=-A(a+k \mu) / \mu, \lambda_{2}=H, \lambda_{3}=b-\theta, \lambda_{4}=-B
$$

Where, $\quad H=(\mu k / \mu k+a)(a-\alpha-a A / \mu k) \quad$ Here, unconditionally $\lambda_{1}<0$ and $\lambda_{4}<0$. However, $\lambda_{2}$ and $\lambda_{3}$ would be negative if the condition $b<\theta$ and $H>1$.

Hence, the equilibrium $E_{3}$ is stable under the stated condition.

Theorem 5: The equilibrium point $\left(0,0, y_{1}{ }^{*}, 0\right)$ is stable if $\left[r k^{\prime}(b-\theta) / b\right]<B$.

Proof:

The variational matrix $M$ at this equilibriumis denoted by $M_{6}$ and given by

$$
M_{6}=\left[\begin{array}{cccc}
a-\alpha & 0 & 0 & 0 \\
0 & -A & 0 & 0 \\
0 & 0 & \theta-b & \frac{-\left(\mathrm{b}+\mathrm{rk}^{\prime}\right)(\mathrm{b}-\theta)}{\mathrm{b}} \\
0 & 0 & 0 & r k^{\prime}(b-\theta) / b-B
\end{array}\right]
$$

The characteristic equation $\operatorname{det}\left(M_{6}-\lambda I\right)=0$ of the variation matrix $\mathrm{M}_{6}$ at the equilibrium $\mathrm{E}_{6}$ takes the form as $(\alpha-a-\lambda)(-A-\lambda)(\theta-b-\lambda) r\left[k^{\prime}(b-\theta) / b\right]-B-$ $\lambda=0$ giving the eigenvalues $\lambda_{1}=\alpha-a, \lambda_{2}=-A, \lambda_{3}=$ $\theta-b, \lambda_{4}=r\left[k^{\prime}(b-\theta) / b\right]-B \quad$. Here, $\lambda_{2}<0$ unconditionally. However, the remaining eigenvalues are conditionally negatives with the conditions

(i) $\lambda_{1}<0$ if $\alpha<a$

(ii) $\lambda_{3}<0$ if $\theta<b$

(iii) $\lambda_{4}<0$ if $r k^{\prime}(b-\theta) / b<B$.

Here, under the considered condition the equilibrium point $\mathrm{E}_{6}$ is stable.

Theorem 6: The equilibrium point $E_{7}\left(x_{1}{ }^{*}, 0,0,0\right)$ is stable if $[\mu k(a-\alpha) / a]<A$.

Proof:

$$
M_{7}=\left[\begin{array}{cccc}
\alpha-a & -(a-\alpha)(a+\mu k) / a & 0 & 0 \\
0 & (\mu k(a-\alpha) / a)-A & 0 & 0 \\
0 & 0 & b-\theta & 0 \\
0 & 0 & 0 & -B
\end{array}\right]
$$

The characteristic equation $\operatorname{det}\left(M_{7}-\lambda I\right)=0$ of the variation matrix $M_{7}$ at the equilibrium $\mathrm{E}_{7}$ takes the form as $(\alpha-a-\lambda)\{[\mu k(a-\alpha) / a]-A-\lambda\}(b-\theta-\lambda)(-B-$ $\lambda)=0 \quad$ giving the eigenvalues $\lambda_{1}=\alpha-a, \lambda_{2}=[\mu k(a-\alpha) / a]-A, \lambda_{3}=b-$ $\theta, \lambda_{4}=-B$. Here, it can be observed that $\lambda_{4}$ is unconditionally negative while the other eigenvalues are negatives conditionally with the following conditions:

(i) $\lambda_{1}<0$ if $\alpha<a$

(ii) $\lambda_{2}<0$ if $\mu k(a-\alpha) / a<A$

(iii) $\lambda_{3}<0$ if $b<\theta$. Based on the set condition, the equilibrium point is stable.

\subsubsection{Global Stability of the System}

To determine the global stability of the system Lyapunov function method is followed in this study.

Theorem 7: The interior equilibrium point 
$\left(x_{1}{ }^{*}, x_{2}{ }^{*}, y_{1}{ }^{*}, \quad y_{2}{ }^{*}\right)$ is globally stable.

Proof:

Construct the Lyapunov function as

$$
\begin{aligned}
& v\left(\begin{array}{llll}
x_{1} & x_{2} & y_{1} & y_{2}
\end{array}\right) \\
& =x_{1}-x_{1}{ }^{*}-x_{1}{ }^{*} \ln \left(x_{1} / x_{1}{ }^{*}\right) \\
& +l\left[x_{2}-x_{2}{ }^{*}-x_{2}{ }^{*} \ln \left(x_{2} / x_{2}{ }^{*}\right)\right] \\
& +m\left[y_{1}-y_{1}{ }^{*}-y_{1}{ }^{*} \ln \left(y_{1} / y_{1}{ }^{*}\right)\right] \\
& +s\left[y_{2}-y_{2}{ }^{*}-y_{2}{ }^{*} \ln \left(y_{2} / y_{2}{ }^{*}\right)\right] \text {. }
\end{aligned}
$$

Where $m, l, s$ are chosen constants.

Now, the differentiation of $v$ with respect to $t$ and after some algebraic manipulation reduces to the form as follow;

$$
\begin{gathered}
\frac{d v}{d t}=\left(\frac{x_{1}-x_{1}{ }^{*}}{x_{1}}\right)\left(\frac{\mathrm{d} x_{1}}{\mathrm{dt}}\right)+l\left(\frac{x_{2}-x_{2}{ }^{*}}{x_{2}}\right)\left(\frac{\mathrm{d} x_{2}}{\mathrm{dt}}\right) \\
+m\left(\frac{y_{1}-y_{1}^{*}}{y_{1}}\right)\left(\frac{\mathrm{d} y_{1}}{\mathrm{dt}}\right)+\mathrm{s}\left(\frac{y_{2}-y_{2}{ }^{*}}{y_{2}}\right)\left(\frac{\mathrm{d} y_{2}}{\mathrm{dt}}\right) \\
=\left(\frac{x_{1}-x_{1}{ }^{*}}{x_{1}}\right) a x_{1}\left(1-\frac{x_{1}+x_{2}}{k}\right)-\mu\left(x_{1} x_{2}\right)-\alpha x_{1} \\
+l\left(\frac{x_{2}-x_{2}{ }^{*}}{x_{2}}\right) \mu\left(x_{1} x_{2}\right)-A x_{2} \\
+m\left(\frac{y_{1}-y_{1}^{*}}{y_{1}}\right) b y_{1}\left(1-\frac{y_{1}+y_{2}}{k^{\prime}}\right)-r\left(y_{1} y_{2}\right)-\theta y_{1}
\end{gathered}
$$

$$
\begin{gathered}
+\mathrm{s}\left(\frac{y_{2}-y_{2}{ }^{*}}{y_{2}}\right) \mathrm{r}\left(y_{1} y_{2}\right)-B y_{2} \\
=\left(x_{1}-x_{1}{ }^{*}\right)\left[\begin{array}{r}
a \\
\left.\left(1-\frac{x_{1}+x_{2}}{k}\right)-\mu x_{2}-\alpha\right] \\
+l\left(x_{2}-x_{2}{ }^{*}\right)\left[\mu x_{1}-A\right]
\end{array}\right. \\
+m\left(y_{1}-y_{1}{ }^{*}\right)\left[\begin{array}{l}
\left.\mathrm{b}\left(1-\frac{y_{1}+y_{2}}{k^{\prime}}\right)-r y_{2}-\theta\right] \\
+\mathrm{s}\left(y_{2}-y_{2}{ }^{*}\right) \mathrm{r}\left[\left(y_{1}\right)-B\right]
\end{array}\right. \\
=-(a / k)\left(x_{1}-x_{1}{ }^{*}\right)^{2}-\mu\left(x_{2}-x_{2}{ }^{*}\right)\left(x_{1}-x_{1}{ }^{*}\right)+ \\
l \mu\left(x_{2}-x_{2}{ }^{*}\right)\left(x_{1}-x_{1}{ }^{*}\right) \\
-\left(b m / k^{\prime}\right)\left(y_{1}-y_{1}{ }^{*}\right)^{2}-m r\left(y_{1}-y_{1}{ }^{*}\right)\left(y_{2}-y_{2}{ }^{*}\right) \\
+\operatorname{sr}\left(y_{2}-y_{2}{ }^{*}\right)\left(y_{1}-y_{1}{ }^{*}\right)
\end{gathered}
$$

\begin{tabular}{|c|c|c|c|c|c|c|c|c|c|c|}
\hline \multirow{2}{*}{ Figures } & \multicolumn{10}{|c|}{ Parameters and their values } \\
\hline & $a$ & $\boldsymbol{b}$ & $\mu$ & $r$ & $\alpha$ & $\theta$ & $\delta$ & $\beta$ & $\boldsymbol{k}$ & $\boldsymbol{k}^{\prime}$ \\
\hline 3 & 1.1940 & 0.2550 & 0.3900 & 3.3920 & 2.4150 & 0.6000 & 2.1600 & 1.0980 & 0.6250 & 2.3920 \\
\hline 4 & 3.1260 & 3.9550 & 0.1600 & 6.2000 & 3.1050 & 0.4800 & 15.2200 & 1.6470 & 0.5450 & 6.9200 \\
\hline 5 & 3.0660 & 2.6200 & 6.0300 & 4.1440 & 8.0700 & 0.4800 & 11.9400 & 2.1960 & 2.6350 & 7.6400 \\
\hline 6 & 5.5380 & 0.8700 & 8.4600 & 1.1040 & 1.7250 & 5.3820 & 1.0400 & 6.0210 & 3.5100 & 1.6240 \\
\hline 7 & 5.5380 & 1.1250 & 8.4600 & 5.0960 & 1.7250 & 5.2680 & 4.4000 & 7.9020 & 3.5100 & 6.0160 \\
\hline 8 & 5.4960 & 1.4950 & 0.2300 & 1.8240 & 0.5850 & 0.2520 & 2.4200 & 3.9060 & 1.2500 & 2.8320 \\
\hline 9 & 5.4960 & 2.5400 & 0.2300 & 3.1600 & 0.5850 & 0.2700 & 2.4200 & 8.0730 & 1.2500 & 5.0640 \\
\hline
\end{tabular}

For simplicity, choosing $l=1$ and $m=s$ the foregoing time derivative reduces to $(d v / d t)=-(a / k)\left(x_{1}-x_{1}{ }^{*}\right)^{2}-$ $\left(b m / k^{\prime}\right)\left(y_{1}-y_{1}^{*}\right)^{2}$. Now, it can be observed that $(d v / d t)<0$

Therefore, the interior equilibrium point is globally stable.

\subsection{Numerical Simulations}

In this section, numerical simulations of the dynamics of the model equation around some of the steady state for certain range of parametric values are used to support the analytical results.

The parameters and its values used in this study are mentioned in the following table as follow;

Table 3. Parameters and their values for figures 3-9.

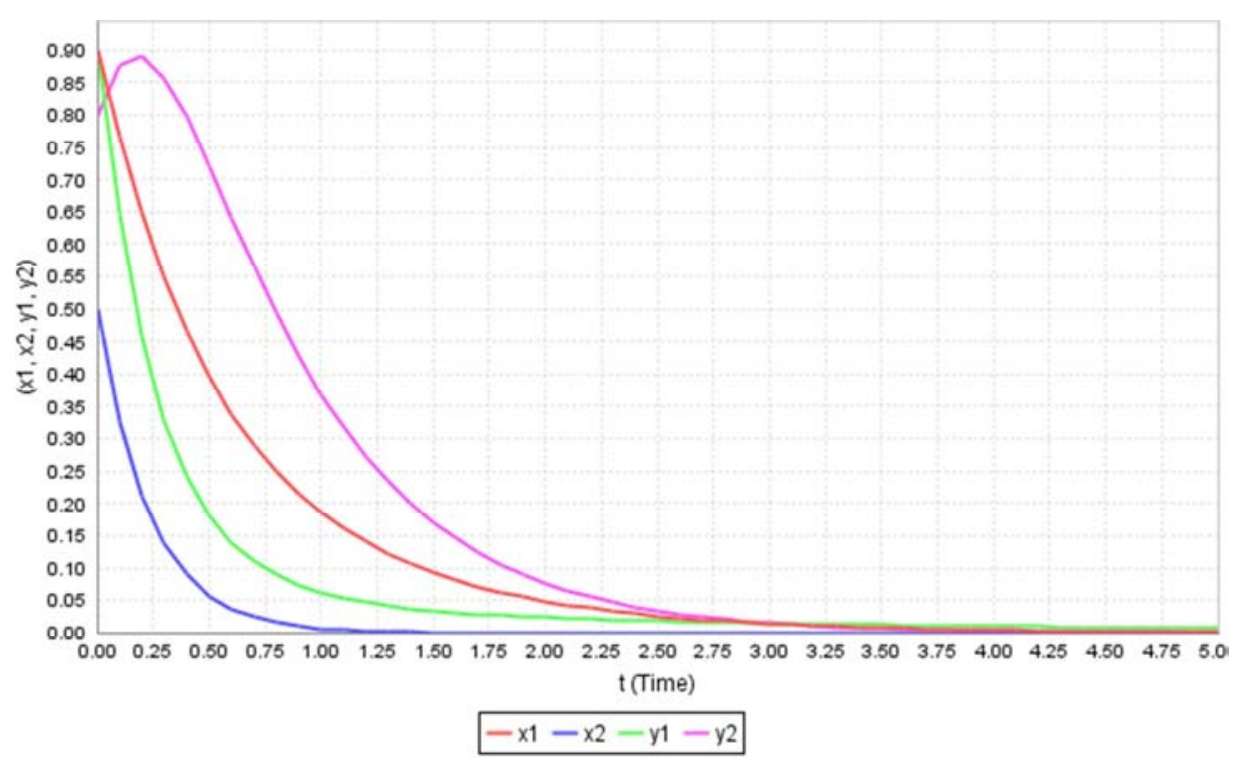

Figure 3. All populations vanish together over time. 
This figure shows that when growth rate of both populations is less than the natural death rate and attacked by disease all the populations become completely extinct.

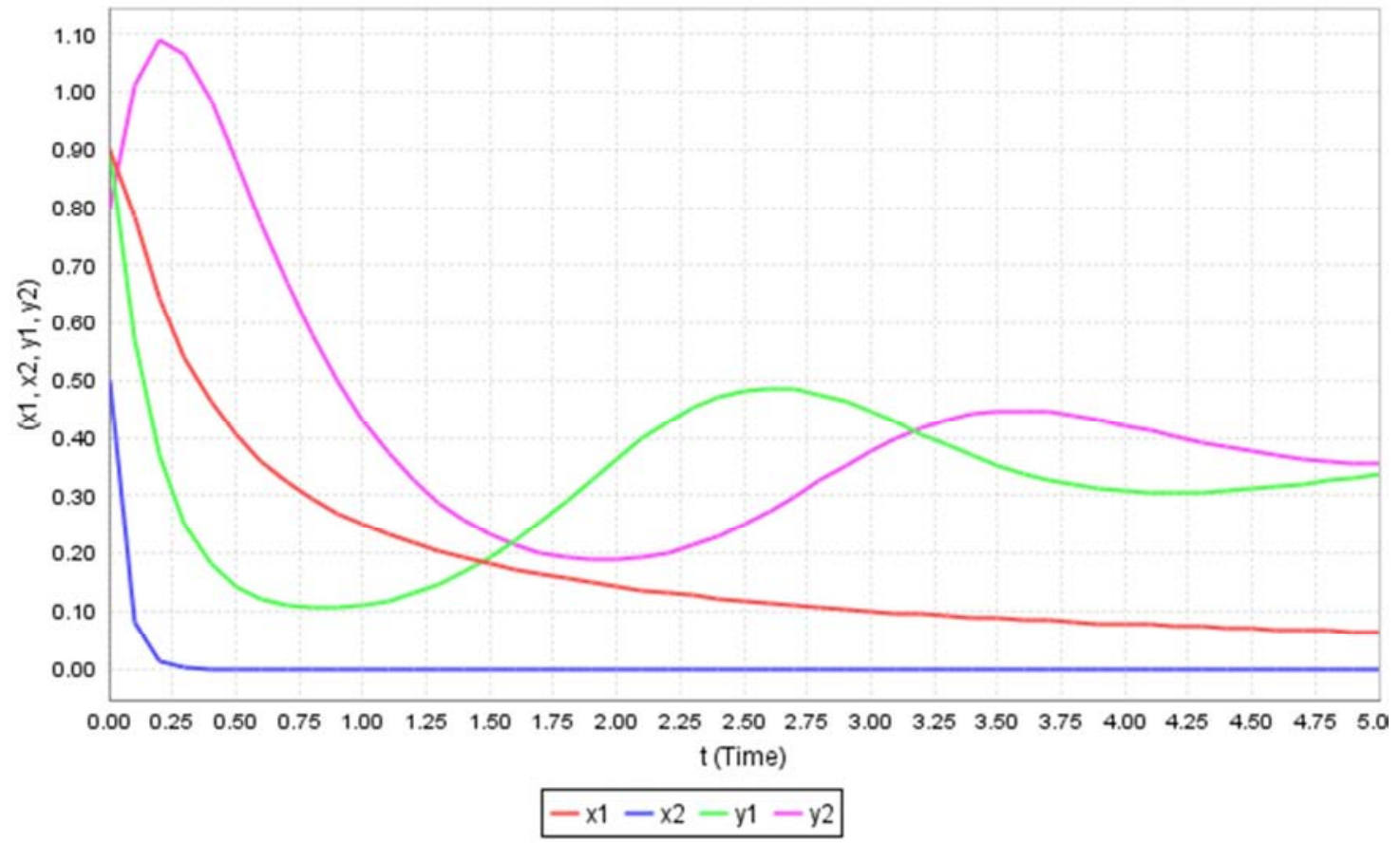

Figure 4. The populations of the second host exist with disease.

This figure shows that the growth rate of host- 2 is greater than its natural death rate and the first host more affected and hence declines more.

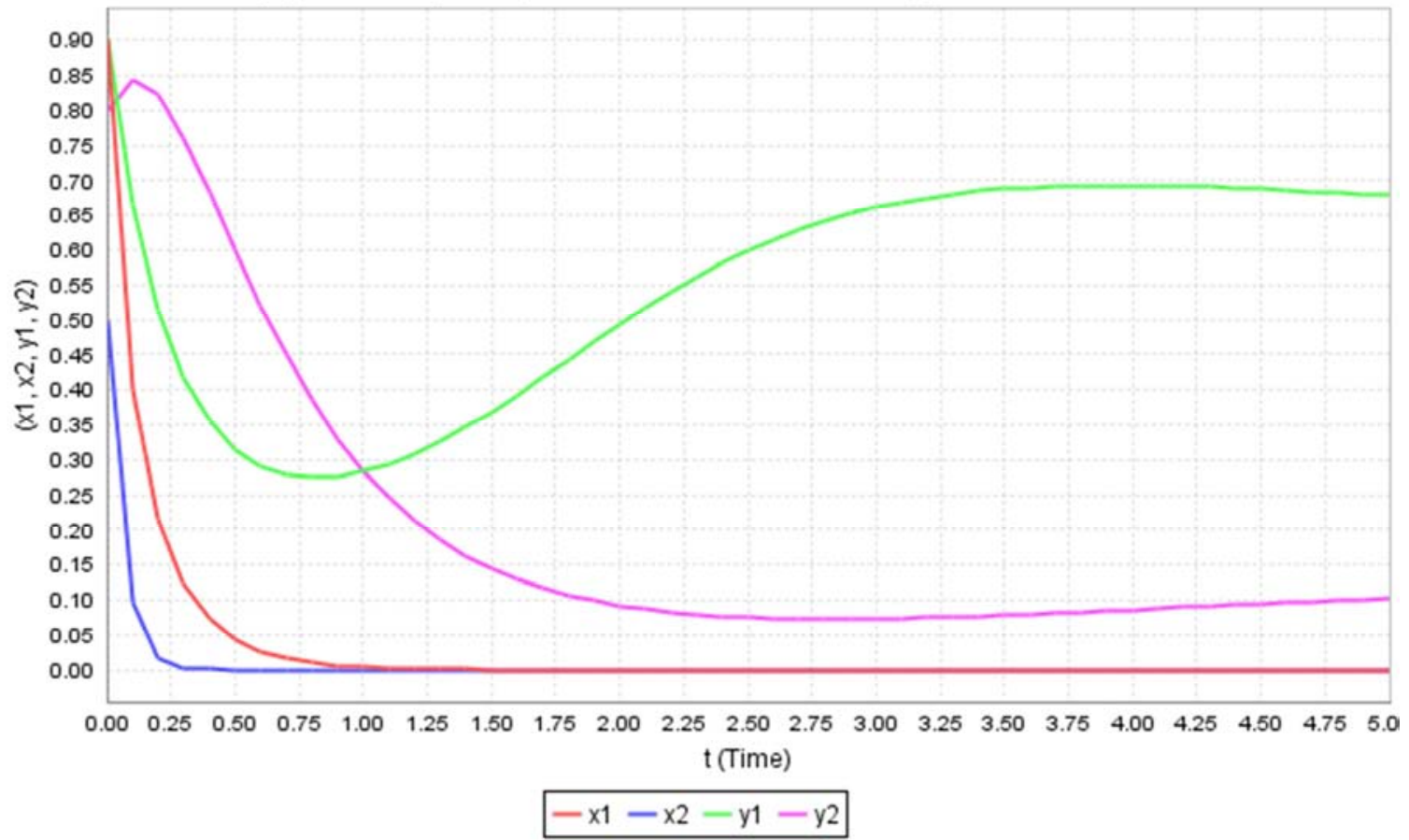

Figure 5. The population of the second host dominant over the other.

This figure shows that the infected host declines more due to the infection rate and natural death rate of host- 1 is increased keeping constant death rate of host- 2 . 


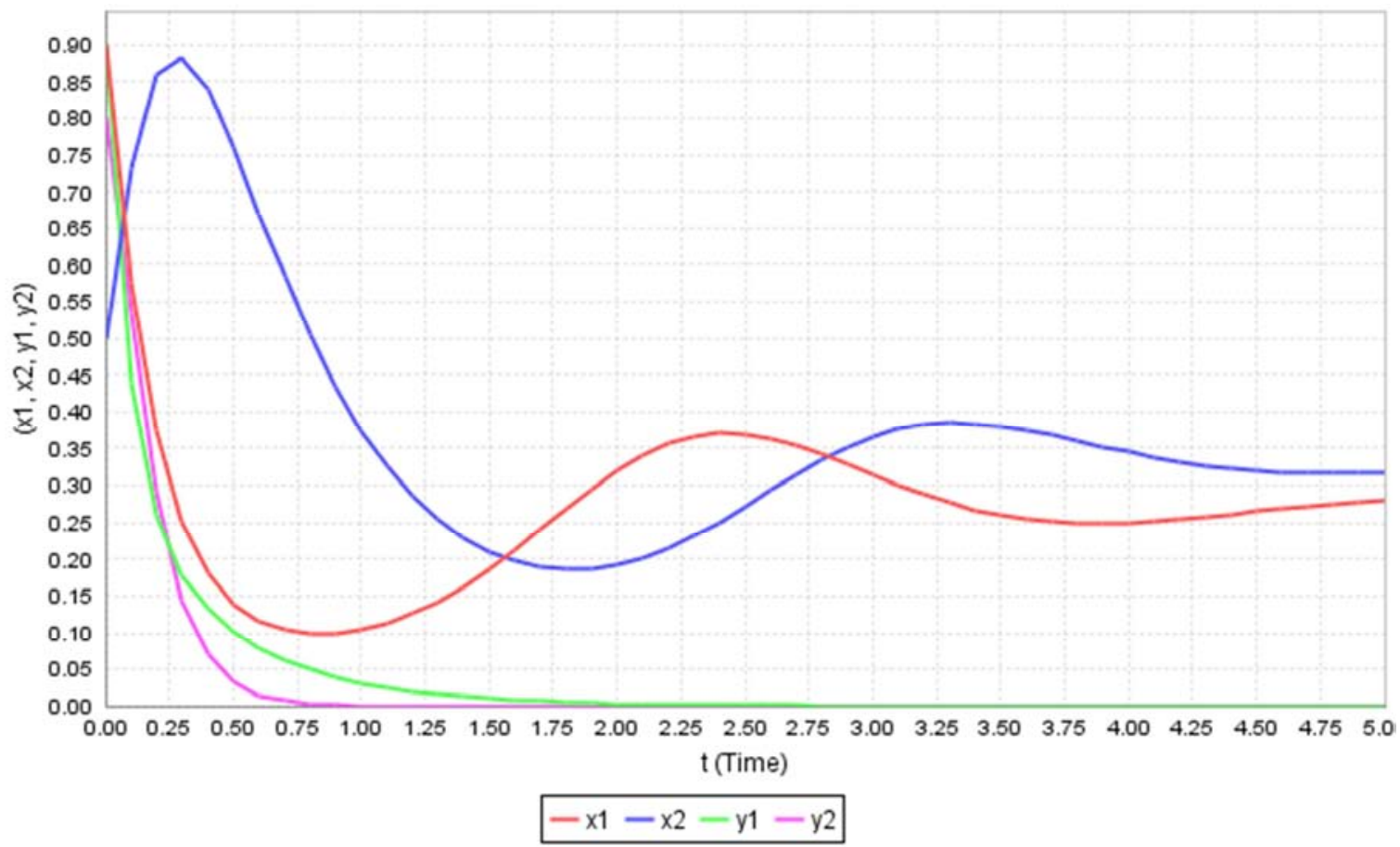

Figure 6. The host population around the equilibrium point $E_{3}$.

One can observe from this figure that host- 2 is more affected by disease and die due to natural death.

But once the populations of the infected starts to decline, the first host become slightly increasing and regulate each other by forcing the second host to extinct without recovery.

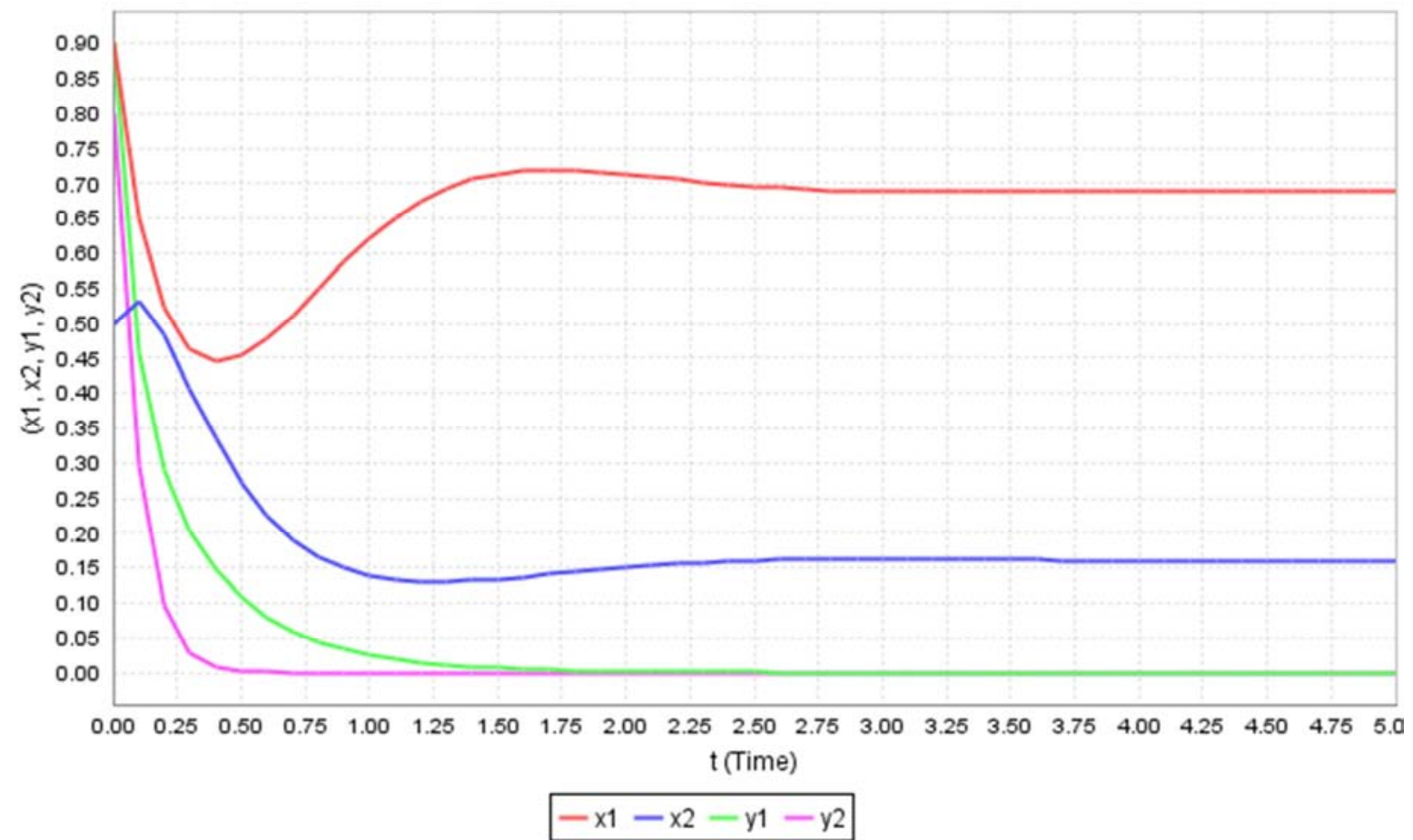

Figure 7. Host-1 population dominant over host-2population.

This figure shows that host-2 is more infected and hence the mortality rate due to disease increases while the first host keeps increasing up to certain time. 


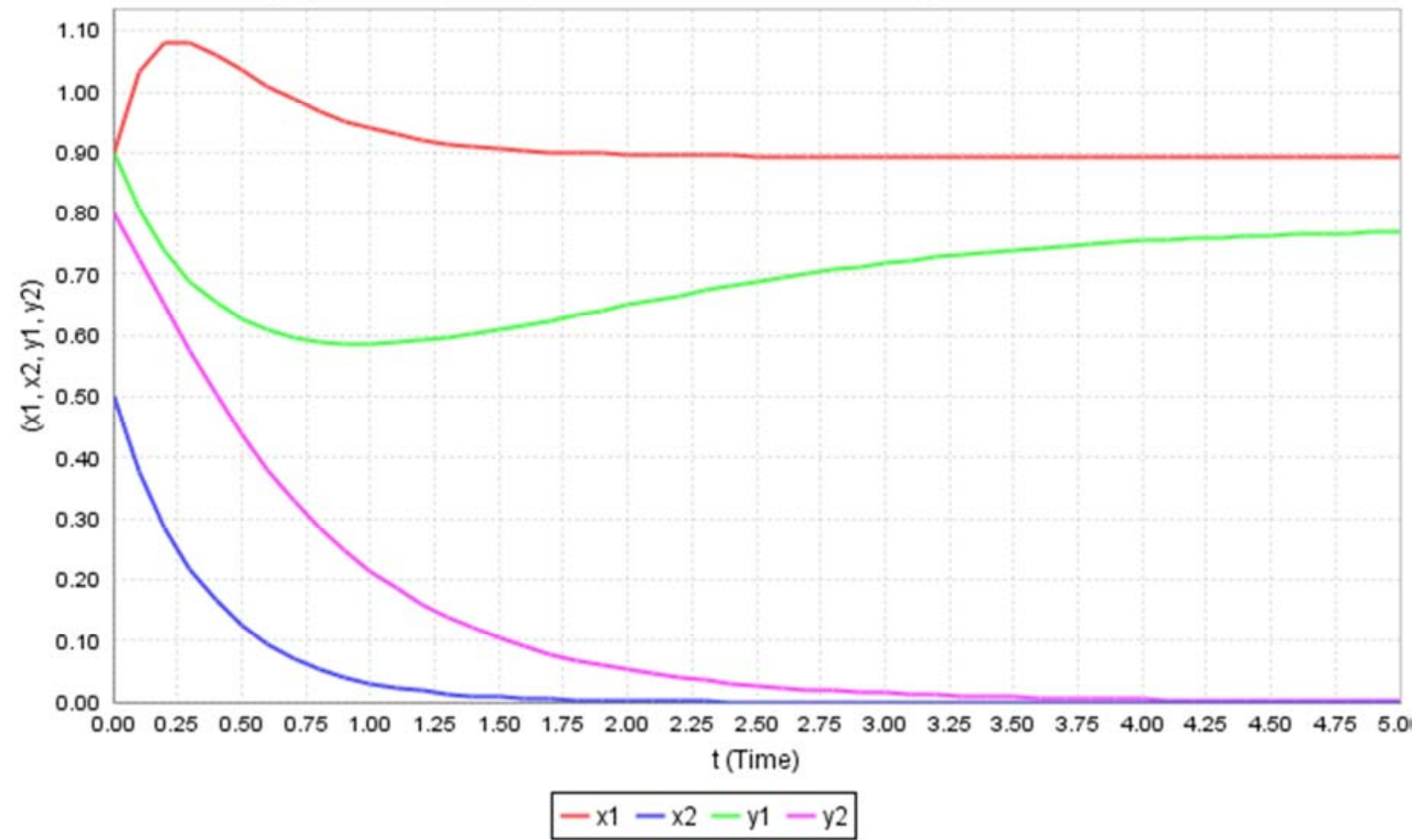

Figure 8. Population dynamics around the equilibrium point $E_{1}$.

This figure shows that both host populations would live together without harming each other when the infected hosts are decline and extinct without recovery.

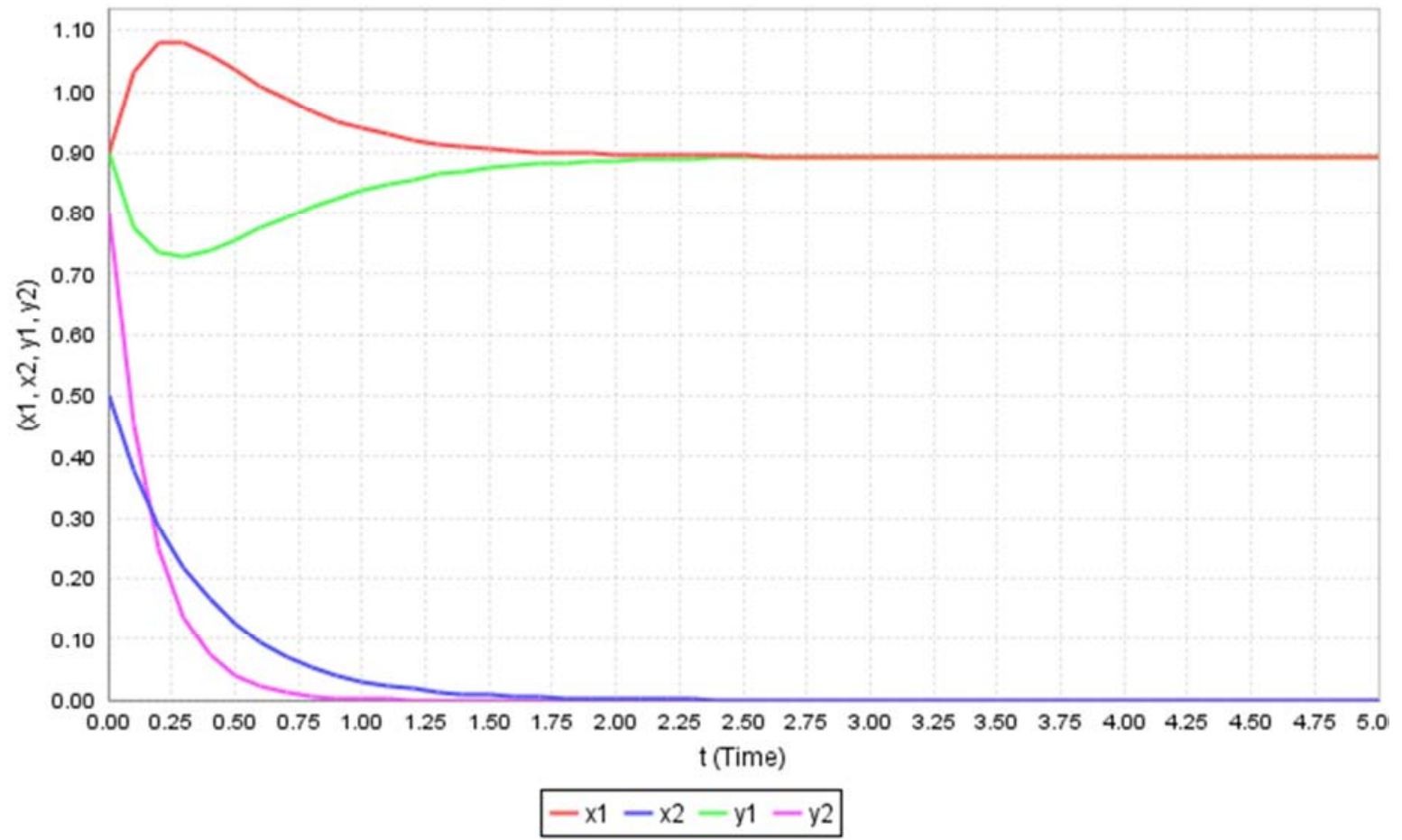

Figure 9. Stabilization of both populations.

This figure shows that when infection transmission rate of host-1and mortality rate due to disease of host- 2 increased keeping the other parameters constant the populations become stabilized.

\section{Conclusion}

In the present study the population dynamics of four dimensional systems has been taken up for investigation. A 
mathematical model for this four dimensional system is developed by studying carefully and incorporating the futures of each system together. The positivity and boundedness of the model variables are verified and hence shown that the developed four-dimensional system is biologically well behaved.

In this paper, species have strong indirect effects on others, and predicting these effects is a central challenge in ecology. Host species sharing an enemy can be linked by apparent competition, that is interaction between two hosts and parasite is carried out for investigation. In the case where infection was permitted, parasite coexists in the population, but only under particular conditions, namely when rates of infection were varies both hosts.

The results from the model system demonstrate that the important role that parasite may play in the establishment of community structures within host populations' dynamics. This was indicated from the stability of interior equilibrium point in which the populations are coexisting.

We need to point out here that, although the model considered in this study is two host populations via shared enemy model. Even though there is no direct interactions specifically associated with the hosts populations, the effects of each host was investigated by altering parameters involved in the system. It has been shown that, this process is strong enough to be a community-wide structuring mechanism that could be used to predict future states of diversification. To show this, numerical study of the model was carried out to support the finding. Moreover, the model system would be fully screened when there is a competition between the hosts that would be mediated by parasite interaction providing shared resources. This would be our next further investigation.

\section{Acknowledgements}

The authors would like to thank the editors and anonymous referee for their valuable comments and suggestions which led to a significant improvement of the work.

\section{References}

[1] AlyssaLois M. (2016). Non-native parasite enhances susceptibility of host to native predators, Springer-Verlag, Berlin, Heidelberg.

[2] Anderson R. M., May R. M. (1981). The population dynamics of micro-parasites and their invertebrate hosts. Phil. Trans. R. Soc. Lond. 291, 451-524.

[3] Anderson, R. and May R. Infectious Diseases of Humans, Dynamics and Control. Oxford University Press, Oxford (1991).

[4] Benjamin J. Z., Buckling A. (2012). The mode of hostparasite interaction shapes co-evolutionary dynamics and the fate of host cooperation. Proc. R. Soc. Lond., B 279, 3742-48.

[5] Bowers R. G., Boots M., Begon M. (1994). Life-history trade- offs and the evolution of pathogen resistance: competition between host strain. Proc. R. Soc. Lond., B 257, 247-53.

[6] Chakra M. A., Hilbe C. and Treutlen A.(2014). Plastic behaviors in hosts promote the emergence of retaliatory parasites, Sci. Rep., 4, 4251.

[7] D. Adak and N. Bairagi. (2014). Dynamical behavior of a predator-prey-parasite model with nonlinear incidence rate, J. Biol. Syst., 1 (1).

[8] Diekmann O., Heesterbeek J. A. P. (2000). Mathematical Epidemiology of Infectious Diseases: Model Building, Analysis and Interpretation. Wiley, New York, p. 365.

[9] Dobson A. P. (2004). Population dynamics of pathogens with multiple host species. Am. Nat., 164, S64-S78.

[10] Fanghong Zhang and CunchengJin. (2017). Analysis of an eco-epidemiological model with Disease in the prey and predator. $6(1), 22-28$.

[11] GeremewKenassa Edessa, BokaKumsa, Purnachandra Rao Koya. (2018) Modeling and Simulation Study of the PopulationDynamics of Commensal-Host-Parasite System. American Journal of Applied Mathematics. Vol. 6, No. 3, pp. 97-108.

[12] GeremewKenassa Edessa, BokaKumsa, Purnachandra Rao Koya (2018). Dynamical behavior of Susceptible preyInfected prey-Predator Populations. IOSR Journal of Mathematics (IOSR-JM) 14.4 PP: 31-41.

[13] Hatcher P. E., Moore J., Taylor J. E., Tinney G. W. and Paul N. D. (2004). Phytohormones and plant-herbivore-pathogen interactions: integrating the molecular with the ecological. Ecology, 85, 59-69.

[14] Hatcher P. E. and Paul N. D. (2000) Beetle grazing reduce natural infection of Rumexobtusifoliusby fungal pathogens, New Phytol, 146, 325-33.

[15] Hatcher P. E., Paul N. D., Ayres P. G. \& Whittaker J. B. (1994). The effect of an insect herbivore and a rust fungus individually and combined in sequence on the growth of two Rumex species. New Phytol, 128, 71-78.

[16] Holt R. D. \& Dobson A. P. (2006). Extending the principles of community ecology to address the epidemiology of host pathogen systems. In: Disease Ecology: Community Structure and Pathogen Dynamics. Oxford University Press, Oxford, pp. 6-27.

[17] Holt R. D. \& Hochberg M. E. (1998). The coexistence of competing parasites. Part II-Hyper parasitism and food chain dynamics. J. Theor. Biol., 193, 485-95.

[18] Holt R. D. (1977) Predation, apparent competition, and the structure of prey communities. Theoretical Population Biology, 12: 197-229.

[19] Rainey P. B. (2002). Antagonistic co-evolution between a bacterium and a bacterio phage. Proc. R. Soc. Lond. B 269, 931-36.

[20] Sand land G. J., Minchella D. J. (2004). Life-history plasticity in hosts exposed to differing resources and parasitism. Can. J. Zool., 82, 1672-77.

[21] Sen P., Das k. (2017). Simultaneous Effects of Prey Defence and Predator Infection on a Predator Prey System. Ann. Bio. Sci., 5 (1): 37-46. 
[22] Siekmann. I., (2013). On competition in ecology, epidemiology and eco - epidemiology. Ecol. Complex, http://dx.doi.org/10.1016/j.ecocom.2013.01.003.
[23] Sule H., MuhamadR., and Omar D. (2014). Parasitism rate host stage preference and functional response of Tamarixiaradiata on Diaphorinacitri. Int. J. Agric. Biol. 16, 783-88. 